\title{
Contribuciones del Zamorano a Latinoamérica en sus Setenta años
}

\begin{abstract}
Simón E. Malo ${ }^{1}$
Resumen. La contribución más importante del Zamorano a la región ha sido en educación agrícola de Aprender Haciendo. Sus graduados son hoy conocidos por su espirito empresarial. Además, sobresalen como profesores, científicos y en el sector público ha habido cientos de ministros de agricultura. En segundo plano, la institución es conocida por haber traído cientos de especies y variedades superiores de plantas para la industria agrícola. Como antiguo explorador de plantas para el gobierno de los Estados Unidos, Wilson Popenoe tenía la experiencia apropiada para tener la visión de lo que seria importante en el futuro de la región. El mejor ejemplo es quizás la palma africana que con su aceite comestible es hoy un enorme renglón en la exportación de muchos países.
\end{abstract}

Palabras clave: Educación agrícola de Aprender Haciendo, introducción de especies, variedades superiores.

\section{Zamorano's Contributions to Latin America in its Seventy Years}

\begin{abstract}
The most significant contribution of Zamorano to the region has been in agricultural education thru learning by doing, and youth professional building, including character formation. Zamorano graduates are today known for their enterprising spirit and passion for doing a job well done. The number of graduates filling key positions in the agricultural industry, teaching and research, as well as the public sector in the region is impressive. The second most important contribution has been the introduction of superior species of plants to the agriculture of the region. As a former plant explorer for the USDA, Wilson Popenoe was ideally qualified for this work based on his experience, contacts, and his vision of what would be important in the future. Perhaps the best example is the African oil palm that today constitutes the core of many export products in the region.
\end{abstract}

Keywords: Agricultural education thru learning by doing, species introductions, superior varieties.

\section{La Enseñanza y Formación a la Juventud}

Sería largo y aburrido el enumerar y comentar sobre todas y cada una de las contribuciones grandes y pequeñas que una institución internacional activa y compleja como el Zamorano ha hecho a la región en sus 70 años de funcionar. Es mejor poner de relieve primero todas sus importantes contribuciones intangibles, es decir, aquellas de conocimientos, conceptos y valores que se van en las mentes de sus graduados después de sus años de residencia en la institución, ya sea que se gradúen o no, pero que son tan difíciles de documentar y medir apropiadamente con precisión. Al enseñar a gente joven es imposible predecir cuan efectivo es el currículo sino hasta años después cuando se ve el efecto de lo que hace el graduado en la vida real de su país.

Desde las primeras graduaciones ha sido muy conocido y aparente en todo Latinoamérica que los graduados Zamoranos han tenido una actitud a la vida y costumbres diferentes, muy sui generis en todas sus actividades cotidianas, pero especialmente singulares en lo que respecta a su trabajo profesional. En todas sus agrupaciones el egresado demuestra una cultura muy característica diferente al proceder de los graduados de otras instituciones agrícolas presentes y pasadas. Su comportamiento diferente va hasta hablar un lenguaje característico o llámemelo con una jerga profesional diversa. Como grupo social, su cohesión entre ellos es notoria y muy reconocida en todos los países de donde vienen. Su adherencia entre ellos es patente y llama la atención de otros grupos que muestran a veces manifestaciones de envidia y aún de una mala disimulada emulación. Al principio en los años ' $40 \mathrm{~s}$ y ' 50 s era conocido en la región el negarles el valor a su diploma, hasta buscando formas estatales

\footnotetext{
${ }^{1}$ Exdirector de la Escuela Agrícola Panamericana, Zamorano, Honduras, 1979-1993. Dirección actual Kansas, Estados Unidos, correo electrónico simbad3033@ hotmail.com
} 
y políticas para desvalorar sus credenciales. Sin embargo esto, increíble ahora, ha desaparecido cuando los Zamoranos son tan abrumadoramente dominantes en la profesión del agro y preferidos sobre otros en el sector agrícola público y privado. En los Estados Unidos, los Zamoranos tienen una extraordinaria demanda y se cree que hay más de 300 graduados entre compañías agrícolas y financieras, en las facultades de universidades y aun en las oficinas estatales de extensión y ayuda al sector agrícola.

Ahora los graduados del Zamorano son reconocidos por su espíritu emprendedor y cooperador, con una pasión por el trabajo duro y en general por su honestidad e integridad individual. Se los identifica por ser los profesionales agrícolas más destacados y por ser los más influyentes en la agricultura de sus países como personas importantes en el campo en donde estén. En los últimos 47 años ha habido un enorme número de Ministros de Agricultura que comenzó en 1965 en la República Dominicana con Ligio A. Tavárez, clase '53. Todos estos han sido muy influyentes con sus políticas eficaces y de espíritu de 'manos a la obra' en la agricultura de sus países.

Las agencias de ayuda internacional (USAID y otros) reconocen de inmediato a los egresados de la Escuela Agrícola Panamericana por su cultura práctica característica, que antes de la fundación de Zamorano no existía en la región. Sería largo y aburrido el describir todo lo que han hecho, pero baste decirlo que hasta en la banca han sobresalido. El Banco del Pacífico, uno de los más grandes del Ecuador y el más técnicamente adelantado fue desarrollado y controlado por Marcel Laniado de Wind (Clase '50) hasta su muerte. Hoy hay cientos de ejecutivos Zamoranos de todo tipo y responsabilidad en todo negocio, especialmente en producción agrícola, exportación: café, cacao, banano, legumbres, tilapia, camarón y otros, programas ambientales de conservación y en especial producción de granos de todo tipo y algodón. Varios cientos se destacan en investigación y enseñanza en universidades por todos lados.

Cuando ya jubilado, Wilson Popenoe en 1965 recibe la noticia del nombramiento de L. Tavárez a Ministro, le manda un telegrama muy especial que el lector tiene que leerlo para que se dé cuenta de lo orgulloso que estaba como Director-Fundador de la
EAP: 'En las palabras del gran Bolívar, ahora puedo bajar a mi tumba en paz, porque había jurado no morir hasta ver a uno de mis muchachos como Ministro de Agricultura.' Yo recibí una copia de este telegrama de Tavárez, ya que fue mi contemporáneo en la EAP.

Estoy seguro que nuestra cultura pragmática nos viene del antiguo Director que hizo lo posible para inculcar e imprimir su propio carácter práctico a la naciente institución que ha perdurado a través de todos estos 70 años y que sus graduados confían que continúe. Esta filosofía y forma de ver las cosas con su valor aplicado es quizá la contribución más sobresaliente que W. Popenoe hizo a Latinoamérica a través de sus estudiantes. Conocía bien que la región tenía una carencia de este enfoque y de ver las cosas en forma utilitaria y práctica. Las deficiencias de nuestra antigua cultura tenía un efecto amenguante en la producción del agro que le faltaba un espíritu eficaz y del ensuciarse las manos, como decía él. Los finqueros antiguos dependían sólo de empleados y peones para hacer las labores manuales y sucias de la agricultura y no querían salir al campo a trabajar. El empeño de Popenoe fue que sus alumnos aprendan desde el primer paso a hacerlo todo personalmente sin depender de nadie. Hizo lo posible por traer profesores/instructores prácticos de todos lados para que mostraran haciendo a los estudiantes y exigieran el hacerlo con las manos. Creo que este aspecto del aprendizaje de manos a la obra y pragmático del Zamorano ha tenido un éxito enorme en la región ya que la EAP es hoy una institución que da el ejemplo por su eficacia en su enseñanza y es aún más aplicada y de manos a la obra que cualquier otra universidad norteamericana, de donde nos vino originalmente esta cultura. Creo que todos los graduados nos sentimos orgullosos de combinar el aspecto pragmático con el científico aplicado en nuestro espíritu y de ser dignos herederos del talento fundamental de W. Popenoe que combinaba estas características en amplia medida.

\section{Introducción de Variedades Nuevas}

El segundo aspecto en las contribuciones del Zamorano, después de la enseñanza y formación a la juventud, es la introducción de variedades nuevas de plantas superiores a la región. Así es que desde los primeros días de la Escuela Agrícola Panamericana, 
Popenoe, con su experiencia de explorador para el Departamento de Agricultura de los Estados Unidos, se empeñó en traer todo tipo de nuevas especies y variedades destacadas a su naciente Escuela. Creía que sus estudiantes debían trabajar con sobresalientes tipos con futuro comercial en la región.

El clima moderado de Zamorano le entusiasmaba mucho, ya que se le parecía al clima subtropical donde él creció en Pasadena, Altadena, en la zona al norte de Los Ángeles, California. Ahora el rol de Popenoe se volvió parecido al trabajo de introducir plantas a Lancetilla para la United Fruit Company. En Zamorano empezó a traer no sólo semillas, sino cientos de nuevas plantas para adaptarlas y probarlas en su valle de mediana altura, y convertirla en una estación experimental técnica, como parte de la enseñanza agrícola. Algunas de estas plantas estaban en Honduras ya que habían sido traídas como posibles reemplazos al banano, que por medio siglo había sido afectado y amenazado por el Mal de Panamá y la variedad dominante, Gros Michel, una vez infectado no tenía remedio.

Con plantas de usos industriales comenzó con palma africana (Elaeis guineensis) con la variedad Del', por su aceite comestible y usos industriales. Esta variedad había sido traída de Sumatra, Indonesia, en 1926 a la costa norte de Honduras por la United Fruit Company, cuando los holandeses todavía ocupaban las islas y eran activos en mejoramiento genético. W. Popenoe creía que sus estudiantes debían conocer bien este cultivo debido a su gran futuro como fuente de aceite comestible. Hoy esta visión insólita al futuro se ha vuelto realidad ya que ahora hay muchos Zamoranos que han hecho respetables fortunas en palma africana. Con este comienzo impulsado por Popenoe y sus colegas de la United Fruit Co., como V.C. Dunlap y M. Trafton, ahora los trópicos americanos se han vuelto grandes exportadores de aceite a China e India, dos países con enorme población y grandes necesidades de aceite comestible. Hoy, compañías como Uniliver en Europa y Procter \& Gamble en los Estados Unidos son los grandes compradores de aceite de palma para el mercado mundial que crece todos los días a grandes pasos debido a la creciente población mundial. Sin embargo, cuando llegué a primer año a Zamorano en marzo, 1951, los primeras cinco acres ( 2 ha) experimentales de Palma ya estaban siendo arrancadas por estudiantes, pero a mí me tocó asimismo aprender que la palma era monoica, de flores unisexuales, con masculinas y femeninas en el mismo pie. "Como el maíz, muchachos", decía Popenoe a los estudiantes.

En pocos años W. Popenoe se había convencido que el clima del Zamorano no servía para la apropiada floración de la palma africana y no iba a producir bien por falta de calor. A $800 \mathrm{~m}$ de altura y 14 grados de latitud norte, las noches son agradables para la gente, pero demasiado frías para la fructificación adecuada y formación del carnoso pericarpio de la fruta en donde está casi todo el aceite de la planta. W. Popenoe no quería desperdiciar buen terreno con escasa agua de irrigación para algo que exigía un clima más caliente. Lo mismo pasó con el pili nut (Canarium ovatum), aunque la línea de árboles que plantó al lado de la palma, a lo largo de una acequia de irrigación, ha producido relativamente bien a través de los años, a pesar del clima que no es el ideal (Figura 1).

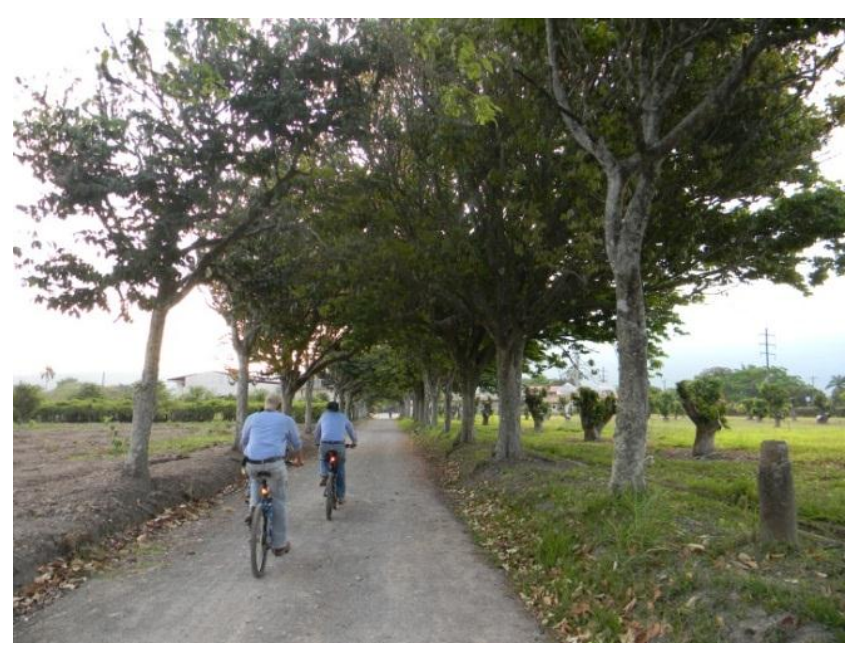

Figura 1. Árboles de pili nut (Canarium ovatum) en la Escuela Agrícola Panamericana, Zamorano, Honduras; este árbol fue llevado a Zamorano por Wilson Popenoe.

Los primeros años de la Escuela Agrícola Panamericana fueron tiempos de intenso trabajo de plantar y establecer nuevas colecciones frutales que vinieron de todos lados. California y Florida, donde Popenoe tenía tantos contactos y amigos, fueron 
fuentes inagotables de nuevas variedades. Todos nos acordamos de los deliciosos mangos de Florida que trajo el Director. Todo estudiante que ha pasado por la Escuela ha probado todos los años el sabroso Pili, la nuez originaria de las Filipinas. Yo personalmente he mandado docenas de paquetes de semilla de Pili a varios países a mis compañeros Zamoranos y quizá sigan produciendo, como en el Ingenio Juan Viñas, cerca de Turrialba, Costa Rica.

En el establecimiento de la Escuela Agrícola Panamericana pronto vinieron docenas de variedades de cítricos, aguacates, guayabas y mirtáceas de muchas especies. Entre las Anonáceas sobresalen las guanábanas (Annona muricata) que los estudiantes usábamos para deliciosos jugos. En las zapotáceas hubo varias especies agradables. Piñas (Ananas comosus) fueron de algunos tipos, especialmente la Cayena Liza que era una delicia para el estudiante. Las jaboticabas (Myrciaria cauliflora) han medrado bien en el Zamorano y durante mi tiempo como instructor, y luego como Director, hicimos lo posible para plantar todas las que pudimos y que ahora son el deleite visual, como planta ornamental, y su fruta es una delicia para el estudiante. Esta fruta como una uva grande es ideal cuando madura y fresca para un estudiante cansado en una tarde caliente y seca. Supongo que solo alguien que ha sido estudiante podrá apreciar plenamente lo que digo. W. Popenoe le dedicó mucho tiempo y entusiasmo a un sinnúmero de especies de legumbre. Por ejemplo, a variedades enormes de buena cebolla (más de 18 tipos), a repollos y a los famosos puerros que tanto le gustaban y que los estudiantes odiaban, ya que exigía que el comedor estudiantil los preparara como a él le gustaban. Todos estos vinieron de Davis, California, traídos por John MacGillivray quien tenía un especial interés en fotoperiodismo de hortalizas en el Zamorano. Todas estas docenas de variedades acabaron en varios países de donde venían estudiantes y visitantes.

W. Popenoe tenía un notable interés por variedades de uva que trajo de varios lugares. Desafortunadamente, ninguna de estas se dieron bien o como él confiaba, por varias enfermedades de la hoja. Niagara' es quizá la que mejor se dio, aunque necesitaba mucho cuido con rociaduras continuas de fungicidas contra enfermedades, especialmente los mildew polvorientos de la hoja. En el monte Uyuca plantó cientos de especies que necesitaban clima templado, frutales y legumbres, cítricos y rosáceas como moras, peras, durazneros, manzanas y otros como los deliciosos Kaki (Diospyros kaki). Aunque muchos no fructificaron bien por la excesiva humedad relativa, sirvieron como fuentes de germoplasma para mandar a cientos de instituciones, cooperadores y colegas de Popenoe por toda la región.

Todas estas especies y variedades nuevas se fueron con los graduados y los estudiantes a una infinidad de lugares por toda la región. Las variedades nuevas de rosas y Cannas de primorosas flores se mandaron por cientos a muchos países y cada vez que las veo en varios lugares me acuerdo de mis días de estudiante e instructor empacando docenas de paquetes de rizomas de Cannas para algún amigo o conocido del Director. Por ejemplo, las rosas y cannas vistosas que se ven en los valles abrigados del Ecuador y Colombia llegaron del Zamorano.

Quizá el mejor ejemplo de una contribución relativamente reciente de Zamorano a la región es la introducción de Ashoka de los trópicos asiáticos. Cuando llegué de Director en enero, 1979, traje conmigo plantas de Polyalthia longifolia, un árbol alto y erecto con una dominancia terminal característica. Es el árbol sagrado ornamental del sur de la India, que yo había llevado a Florida desde Tailandia, pensando en Zamorano como destino final. Florida, aún en Homestead en el mero extremo sur del estado, no es apropiado para este árbol por sus ocasionales devastadoras heladas. Esta Annonacea ornamental se ha dado tan bien en Zamorano a lo largo de avenidas como árbol ornamental, que es una atracción llamativa al visitante (Figura 2). La semilla la han distribuido los estudiantes y visitantes por todo los trópicos americanos. Yo he visto Ashoka en todos lados del Perú amazónico y los trópicos mejicanos. Que yo sepa, todas vienen de las 30 semillas que compré en un vivero en Bangkok en 1978 y que me costaron la ridícula suma de 20 dólares, pero que la mujercita creyó que se había sacado la lotería. Fue una gran ganga porque son tan difíciles de conseguir y los asiáticos son muy celosos con esta planta. 


\section{Publicaciones}

Hablando de contribuciones, Popenoe sabía del interés del público regional por nuevos tipos de frutales y en enero, 1952, publica en la revista Ceiba, la revista periódica de Zamorano, un librito de 367 páginas. Fue publicado primero en inglés como Central American Fruit Culture (Popenoe 1952) y es traducido al castellano y aparece el año siguiente febrero, 1953, como Fruticultura Centroamericana (Popenoe 1953). La traducción fue hecha por Juvenal Valerio, exprofesor de biología de la Escuela, que estaba con el Instituto Interamericano de Ciencias Agrícolas en Turrialba, Costa Rica. Fue revisado por Paul Standley y otras personalidades en la botánica regional y contiene los dibujos originales de Albert Frey hechos para el botánico B. F. Dahlgren del Chicago Natural History Museum. Fue y ha sido un libro enormemente útil y una contribución espléndida a la región que vino a complementar su libro de 1920: Manual of Tropical and Subtropical Fruits (Popenoe 1920) Infelizmente pocos ahora conocen ambos porque son difíciles de conseguir y el primero de 1920 nunca fue traducido al español.

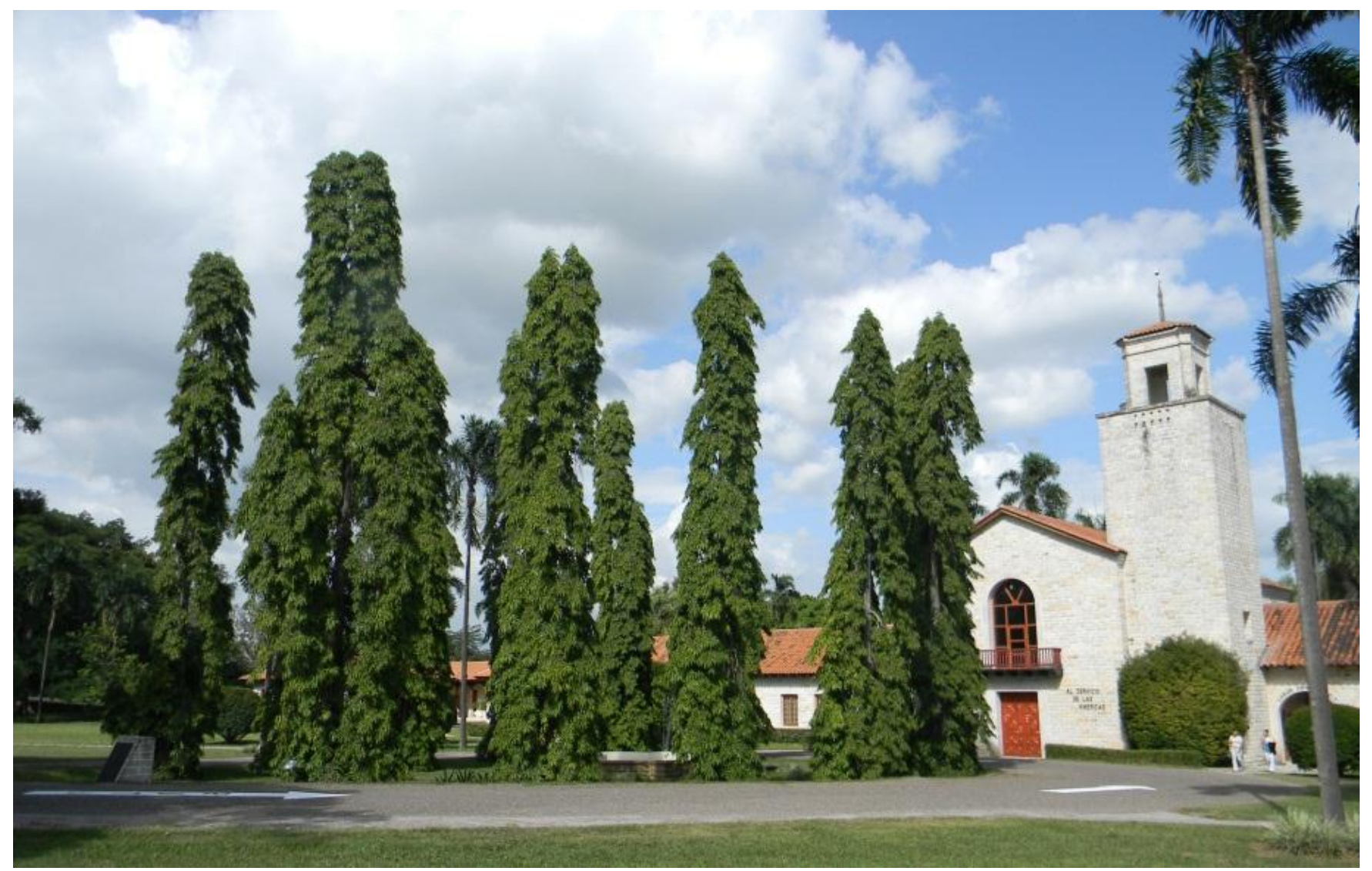

Figura 2. Árboles de Polyalthia longifolia, especie introducida por Simón E. Malo en 1979, al frente del edificio Zemurray en la Escuela Agrícola Panamericana, Zamorano, Honduras, en el 2012.

Otra publicación importante que salió del Zamorano, de las muchísimas, fue en el año 1966 cuando Francisco de Sola organizó un simposio internacional sobre la introducción de plantas en honor a Wilson Popenoe. Ya el agasajado tenía 74 años y no estaba en su mejor salud. De Sola como Presidente de la Junta Directiva de la Escuela Agrícola Panamericana pudo conseguir de la Fundación 
Rockefeller fondos para traer invitados prominentes de varios países, gente que habían trabajado y cooperado con Popenoe en su rol de explorador y de identificar plantas útiles. Hubo 21 artículos sobre todo aspecto de introducción de plantas y obviamente Popenoe estuvo muy contento de ver su labor reconocida con tanta atención por personas amigas y prominentes. Fueron a visitar Lancetilla, que todavía estaba en manos de la United Fruit Co. y no bajo el cuido del Ministerio de Agricultura de Honduras como ahora. Es una publicación enormemente importante que todo graduado de la Escuela Agrícola Panamericana debe leer.

Lista de artículos:

- The History of Plant Exploration and Introduction in the US Department of Agriculture: Knowles A. Ryerson.

- The role of Plant Introduction Gardens: John Popenoe.

- Plant Introduction Gardens in the Caribbean: John V. Walkins.

- $\quad$ The United Fruit Company and the Lancetilla Experiment Station: V. C. Dunlap.

- The Role of Botany and Herbaria in Plant Introduction: Louis O. Williams.

- The Mechanics of Plant Introduction: Harold F. Winters.

- $\quad$ Plant Quarantine Aspects of Plant Introduction: Robert P. Kahn.

- Avocado Introduction in California: C. A. Schroeder.

- The Climatic Adaptability of Pome and Stone Fruit Culture: R. J. Ticho.

- Plant Introduction as Related to Roots and Tubers: Jorge León.

- Plant Introduction Relating to Potatoes: Donovan S. Correll.

- Plant Introduction as Related to Small Grains and Rice: H. A. Rodenhiser.

- Plant Introduction as Related Corn and Sorghum: E. C. Johnson.

- Introduction of Grasses and Legumes for Forage-Problems and Opportunities: A. A. Hanson.
- $\quad$ Recent Introductions of Wild Arabica Coffee from Ethiopia for Updating Coffee Research: F. G. Meyer

- The Development of the Macadamia Nut Industry in Hawaii- A Sequel to Plant Introduction: Ed. T. Fukunaga.

- $\quad$ Plant Introduction with Theobroma cacao. Ernest P. Imle and José Cuatrecasas.

- Three Phases in Vegetable Introduction: Ernesto H. Cásseres.

- Handling and Storage of Plant Materials and Seed: Harold F. Winters.

- Economic Impact of Plant Introduction in Latin America: Jesús Patiño Navarrete.

De todos los 21 artículos presentados, francamente el que más me gusta es el escrito por Knowles Ryerson, el compañero de infancia de Wilson Popenoe. Es un artículo que satisface por lo detallado y completo. Yo tuve la suerte de conocer al Dr. Ryerson cuando él ya era el Decano Emérito de la Universidad de California, Berkeley. Era un hombre bondadoso con los jóvenes y con una mente clarísima para todo. Popenoe lo trajo a mi oficina en Homestead para presentarle a uno de sus antiguos estudiantes. Yo era entonces el miembro más joven de la facultad de la Universidad de Florida en la Estación Subtropical de Homestead. Nunca me olvidaré lo que me dijo, que yo estaba en un lugar privilegiado por su clima para trabajar con plantas tropicales, que cuando él había sido joven, recién abriéndose paso en la profesión, había aplicado para una posición en Homestead, que Wilson Popenoe le había aconsejado que lo hiciera. Ambos decían que básicamente tenían una especial fe en el futuro de Latinoamérica, en todo tipo de su agricultura y hablamos del futuro del Zamorano y el de sus graduados en la región. Mostraba, como profesor en California, una especial generosidad de espíritu con los jóvenes. Se conocía con Popenoe desde que eran niños y vecinos en Altadena, California. En las vacaciones de verano salían en sus bicicletas destartaladas a buscar árboles de aguacate, que eran una rareza en esos tiempos allá. Aquí también se quedaba a dormir en la misma cama con Wilson, quien decía riéndose: "Y nunca me dejaba 
dormir porque me pateaba toda la noche". Ambos tenían un característico humor con un toque burlón. Ryerson había sido el sucesor de David Fairchild cuando este se jubiló en 1928 como Jefe de la célebre Oficina de Seed and Plant Introduccion, creada en Washington en 1898 por el USDA a insistencia de Fairchild. Así que conocía toda la historia del viejo personal y de todo el recorrido de Wilson como explorador de plantas. Popenoe había pasado por esta influyente oficina de 1916 a 1925, fecha que va a Honduras a establecer el Jardín Experimental de Lancetilla para la United Fruit Co. Ryerson describe con entusiasmo lo que tanto hizo Fairchild en esta oficina por su país trayendo del mundo entero las mejores especies y variedades agrícolas para la mejoría de la agricultura. Así que Wilson, a través de su jefe antiguo, sabía cuales eran las más selectas especies para traer a Zamorano.

Otro artículo que tiene mucho que ver con introducción de plantas a Zamorano y con Wilson Popenoe es uno escrito por V.C. Dunlap, en un tiempo el Director de investigación de la Compañía United Fruit Company. El autor relata con autoridad todo lo que se hizo en este Jardín Botánico. Como viejo colega de Wilson Popenoe, que lo conocía bien y sabía lo que había hecho, lo cuenta con autoridad y como el mejor testigo. Comenzando en 1925 se inició este jardín como respuesta a la alarma creciente que tenían los ejecutivos de la compañía por el avance el Mal de Panamá en la región y la destrucción de miles de hectáreas de plantaciones de banano variedad Gros Michel. La principal idea era traer y probar todas las posibles plantas comerciales de reemplazo para el banano. En este punto el mismo Popenoe estaba de acuerdo que la mejor introducción fue la palma africana que después se cultivó en varios países comenzando en Honduras, en San Alejo, y ahora diseminado por todos lados con el clima apropiado.

Por supuesto, mucho de lo anterior no fuera posible sin que Sam Zemurray hubiera dado el primer paso fundando la Escuela Agrícola Panamericana en 1942, y ayudando con su dinero para dar una oportunidad a todos los estudiantes y agrónomos de hoy que han hecho tanto por la región en estos 70 años. Esto también incluye una apreciación por lo antiguo, lo histórico y el valor antropológico que nos han dejado de legado su hija Doris Zemurray Stone (Malo, 1994) y el mismo Popenoe con la preservación de la famosa Casa del Oidor en Antigua Guatemala, Guatemala.

Otras referencias sobre las contribuciones de la Escuela Agrícola Panamericana a la región son las siguientes: MacGillivray, 1956; Popenoe, 1956; Trafton, 1951; Stone, 1954 y Williams, 1981.

\section{Literatura Citada}

Malo, S.E. 1994. Doris Zemurray Stone 1909-1994. Ceiba 35(1):1-2.

MacGillivray, J.H. 1956. El Efecto de algunos factores agronómicos sobre la producción de legumbres en Honduras. Ceiba 4(6):359

Popenoe, W. 1920. Manual of Tropical and Subtropical Fruits. The Macmillan Company, New York.

Popenoe, W. 1952. Central American fruit culture. Ceiba 1(5):269-367.

Popenoe, W. 1953. Fruticultura Centroamericana. Ceiba $3(4): 225-338$.

Popenoe, W. 1956. Tribute to Florida. Ceiba 6(6):309-314.

Stone, D. 1954. Estampas de Honduras. Impresora Galve, S.A. México.

Trafton, M. 1951. The African oil palm in Honduras. Bull. No. 2 of the United Fruit Co. Tropical Research Dept. La Lima, Honduras.

Williams, L.O. 1981. The useful plants of Central America. Ceiba 24(1-2):1-342.

Recibido para publicación el 2 de julio de 2012.

Aceptado para publicación el 30 de noviembre de 2012. 